\title{
Human $\beta$-defensin 2 expression in ELBW infants with severe necrotizing enterocolitis
}

\author{
Andreas C.W. Jenke', Matthias Zilbauer ${ }^{2}$, Jan Postberg ${ }^{1}$ and Stefan Wirth ${ }^{1}$
}

BACKGROUND: The aim of this study was to analyze whether the mucosal innate immune response of extremely-low-birthweight (ELBW) infants might play a role in the development of necrotizing enterocolitis (NEC).

METHODS: Between April 2008 and December 2009 antimicrobial peptides were prospectively measured in fecal samples of ELBW infants. In cases requiring abdominal surgery, fullthickness gut biopsies were analyzed for expression of human $\beta$-defensin 2 (hBD2), interleukin-8 (IL-8), villin, MD2, and Tolllike receptor 4 (TLR4).

RESULTS: Fecal hBD1 concentrations were consistently low in all patients, whereas hBD2 concentrations were high in meconium, particularly in clinical chorioamnionitis, and then dropped, followed by a steady increase after day 14. Infants with moderate NEC showed significantly increased fecal hBD2 concentrations before clinical symptoms, in contrast to infants developing severe NEC. Analysis of intestinal resection material obtained from patients with severe NEC revealed low hBD2 mRNA and protein levels, and increased expression of the inflammatory cytokine IL-8.

CONCLUSION: High hBD2 concentrations, reflecting strong intestinal immune responses, were associated with moderate courses of the disease. In severe NEC, low hBD2 expression was accompanied by low TLR4/MD2 expression, suggesting an inadequate response to luminal bacteria, possibly predisposing those infants to the development of NEC.

T he innate and adaptive immune systems of extremely-lowbirth-weight (ELBW) infants are known to be immature, including deficiencies in neutrophil maturation and migration (1), deficient immunoglobulin production, and complement synthesis (2). Therefore, ELBW infants are generally highly susceptible to systemic infections and necrotizing enterocolitis (NEC), a potentially lethal condition with an overall mortality of $22-62 \%$ in this age group $(3,4)$. Furthermore, in ELBW infants, the gastrointestinal tract is particularly susceptible to aberrant postnatal bacterial colonization, and prolonged antibiotic treatment can result in a paucity of bacterial species, with a relative predominance of Gram-negative bacteria as compared with healthy term infants (5). An important part of the mucosal innate host defense consists of a group of evolutionarily conserved antimicrobial peptides including human $\beta$-defensins (hBDs) (6). Dysregulated expression of these peptides has been implicated in disease pathogenesis of several intestinal inflammatory conditions, including chronic inflammatory bowel diseases (7). Two of the most extensively studied members of this peptide family are hBD1 and hBD2. Under physiological conditions, hBD1 is expressed constitutively by intestinal epithelial cells, whereas hBD2 expression was found to be induced during infection and inflammation. hBD2 exhibits potent bactericidal activity against Gram-negative bacteria and eukaryotic pathogens, such as Candida strains (8), and reduced expression has been observed previously in preterm infants as compared with full-term babies (9); the expression of hBD2 is partly dependent on Toll-like receptor 4 (TLR4) (10), which is mainly stimulated by lipopolysaccharide (LPS), the glycolipid component of the outer membrane of Gramnegative bacteria. For correct function of TLR4, in vivo binding of MD2, a 20-30-kD glycoprotein secreted by endothelial and epithelial cells to its extracellular domain, is essential (11).

Of note, recent evidence obtained from a rodent model of NEC led to the speculation that overstimulation of TLR4 by LPS may be an important factor contributing to NEC development in human ELBW infants (12). However, data obtained from human studies do not support these speculations. In fact, Wolfs et al. recently showed that MD2 was completely absent in healthy and inflamed premature guts. The authors concluded that the inability to sense LPS rather than an over-reaction to it might predispose ELBW infants to NEC (13). In addition, TLR4 levels were found to be significantly lower in monocytes of ELBW infants as compared with healthy term infants, leading to an impaired cytokine production on LPS stimulation (14). Currently, it is therefore unclear whether overstimulation, immaturity, or dysregulation of the immune system is responsible for an increased risk of NEC in premature ELBW infants.

Our study aimed to assess the intestinal mucosal innate immune response of otherwise healthy ELBW infants as compared with infants who went on to develop NEC and to determine whether hBD2 might be a useful disease predictive marker. Of note, in contrast to earlier studies, we monitored

\footnotetext{
The first two authors contributed equally to this work.

'Department of Neonatology, HELIOS Children's Hospital, Witten/Herdecke University, Wuppertal, Germany; ${ }^{2}$ Department of Paediatric Gastroenterology,

Addenbrooke's Hospital, Cambridge, UK. Correspondence: Andreas C.W. Jenke (andreas.jenke@helios-kliniken.de)
} 
the intestinal innate immune response prospectively and independently of clinical condition by measuring the concentrations of hBD1, hBD2, and calprotectin as additional markers for intestinal inflammation (15) in fecal samples of ELBW infants. Those infants who went on to develop NEC and required surgical intervention had levels of hBD2, VIL1, interleukin-8 (IL-8), TLR4, and MD2 mRNA determined in distal small-bowel resections and, if available, at term during stoma closure. Overall, our analyses provide novel insight into the development of the intestinal innate immune system as well as potential implications for NEC disease pathogenesis.

\section{RESULTS}

\section{Epidemiological Characteristics}

During the study period, 78 ELBW infants were admitted. Prevalence of NEC was $19.2 \%(n=15)$. Ten patients were excluded from the analysis due to comorbidities (see Methods section), among those three infants with NEC. Among the remaining 68 ELBW infants, six were diagnosed with severe NEC, six with moderate NEC, and 56 had no gastrointestinal disorder (Table 1). Five infants with severe NEC had preceding intestinal pneumatosis or portal venous gas before pneumoperitoneum. One patient presented exclusively with radiological signs of severe gaseous abdominal distension. No significant differences between groups were observed regarding birth weight, gestational age, $\mathrm{pH}$ and C-reactive protein at birth, and APGAR at $5 \mathrm{~min}$. However, patients with NEC tended to receive longer early antimicrobial treatment and to be less often fed human breast milk as compared with healthy controls (Table 1). All patients with severe NEC required surgery, whereas no surgical intervention was carried out in any other patient group. Mortality rate of severe NEC was $66 \%(n=4)$ with no further deaths in the remaining patients. A total of 732 fecal samples were available (mean 11 per individual, range 5-14).

\section{Fecal hBD1 and hBD2 Concentration in Meconium}

First, we analyzed hBD1 and hBD2 peptide levels in meconium from all ELBW infants and found levels to range from 9.0 to $189 \mathrm{pg} / \mathrm{g}(\mathrm{hBD} 1)$ and 61 to $1,102 \mathrm{ng} / \mathrm{g}$ (hBD2). No significant differences for either hBD1 or hBD2 were observed between the groups. However, ELBW infants born with clinical chorioamnionitis $(n=24)$ had significantly higher concentration of hBD2 in their meconium (mean $704 \mathrm{ng} / \mathrm{g}$, range $473-1,102 \mathrm{ng} / \mathrm{g}$ ) as compared with infants with no evidence of infection $(n=44$; mean $292 \mathrm{ng} / \mathrm{g}$, range $61-589 \mathrm{ng} / \mathrm{g}, P<$ $0.0001)$. In contrast, no difference was noted for hBD1 levels.

Fecal hBD1 and hBD2 Concentrations in ELBW Infants Without Gastrointestinal Disorders

Investigating fecal hBD1 and hBD2 levels, we observed a significant decrease in fecal hBD1 in healthy ELBW infants, with postnatal levels dropping down to a baseline concentration of $10.6 \mathrm{pg} / \mathrm{g}(6.3-18 \mathrm{pg} / \mathrm{g})$ (Figure 1a). A similar effect was observed for fecal hBD2, which fell to a nadir of $36 \mathrm{ng} / \mathrm{g}(5-109 \mathrm{ng} / \mathrm{g})$ at day 14. However, in contrast to hBD1, following the initial decrease we observed a steady increase of hBD2 to an average level of $71 \mathrm{ng} / \mathrm{g}(31-123 \mathrm{ng} / \mathrm{g}, P<0.001)$ at day 28 (Figure $\mathbf{1 b}$ ).

We did not observe any effect of the amount of enteral feeds on fecal hBD1 or hBD2 concentrations, nor did concentrations differ in patients receiving breast milk (mean hBD2 $74 \mathrm{ng} / \mathrm{g}$ at day 28) as compared with those receiving formula feeding (mean hBD2 $70 \mathrm{ng} / \mathrm{g}$ at day 28). In addition, in contrast to our findings in meconium samples, fecal hBD2 concentrations did not differ between patients born with and without clinical chorioamnionitis or in infants subsequently developing moderate or severe NEC until onset of NEC.

\section{Lack of hBD2 Expression in the Inflamed Mucosa of ELBW Infants With Severe NEC}

Having demonstrated a significant postnatal drop of intestinal hBD2 expression in healthy ELBW infants, we next analyzed fecal hBD1 and hBD2 levels in relation to severity of NEC development. Levels of fecal hBD2 increased significantly 12 to $72 \mathrm{~h}$ before clinical signs in patients developing moderate NEC $(183 \mathrm{ng} / \mathrm{g})$, whereas no increase was seen in patients with severe disease ( $42 \mathrm{ng} / \mathrm{g}$, Figure 1c). Of note, in infants with severe NEC, fecal hBD2 concentrations remained within the same range as in the reference population, even at the clinical

Table 1. Epidemiological characteristics

\begin{tabular}{|c|c|c|c|}
\hline Characteristics & $\begin{array}{l}\text { Group with severe NEC } \\
\qquad(n=6)\end{array}$ & $\begin{array}{l}\text { Control group with NEC stage } 2 \\
\qquad(n=6)\end{array}$ & $\begin{array}{l}\text { Control group without any } \\
\text { Gl problems }(n=56)\end{array}$ \\
\hline Gestational age & $25.1(24-27)$ & $25.9(24.8-26.8)$ & $25.3(23.3-27)$ \\
\hline Birth weight in grams & $694(650-745)$ & $800(650-890)$ & $751(354-990)$ \\
\hline $\mathrm{pH}$ at birth & $7.34(7.26-7.44)$ & $7.34(7.27-7.45)$ & $7.32(7.23-7.45)$ \\
\hline C-reactive protein at birth in $\mathrm{mg} / \mathrm{dl}$ & $2.09(0.85-4.32)$ & $0.94(0.3-2.41)$ & $1.67(0.2-4.64)$ \\
\hline Oral feeding at day $7 \mathrm{in} \mathrm{ml} / \mathrm{kg}$ & $98.9(55-142)$ & $85.8(50-138)$ & $84.9(50-168)$ \\
\hline Parenteral fluids at day 7 in $\mathrm{ml} / \mathrm{kg}$ & $74.2(21-146)$ & $81.0(32-140)$ & $78.8(0-128)$ \\
\hline Days on antibiotics during 1 st week & $5.4(4.0-7.0)$ & $5.8(4.2-7.0)$ & $4.4(2.2-6.8)$ \\
\hline Mean onset of NEC (postnatal day) & $17(8-28)$ & $12.5(8-17)$ & - \\
\hline$\%$ Of breast milk feeding $(n)$ & $16.7(1)$ & 0 & $21.4(12)$ \\
\hline$\%$ Of female $(n)$ & $50(3)$ & $50(3)$ & $58.9(33)$ \\
\hline
\end{tabular}

Data are expressed as the mean \pm 2 SD.

$\mathrm{Gl}$, gastrointestinal; NEC, necrotizing enterocolitis. 
a

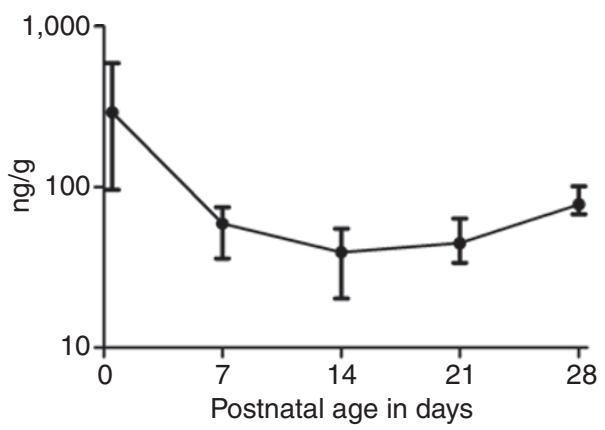

C

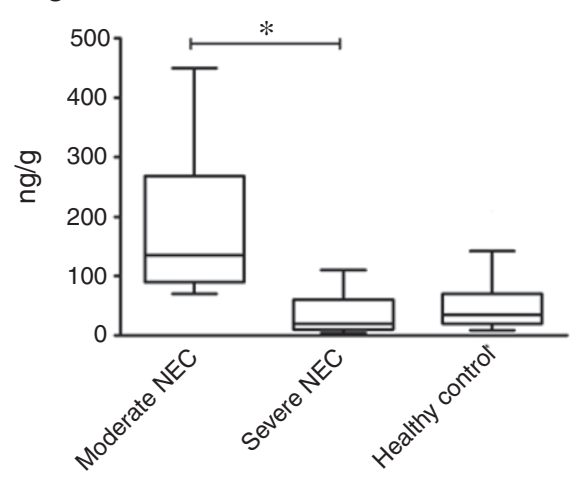

\section{e}

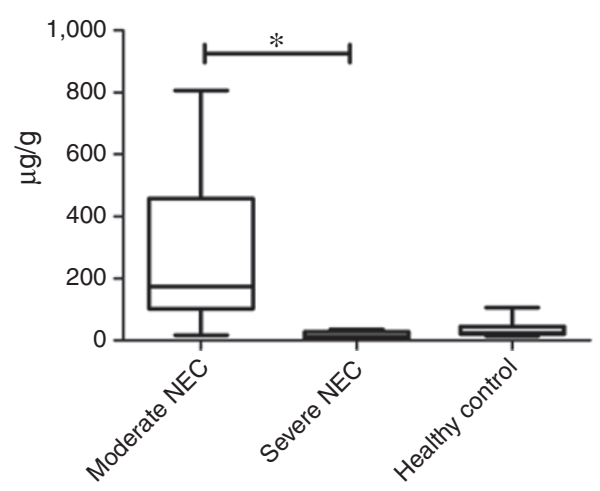

b

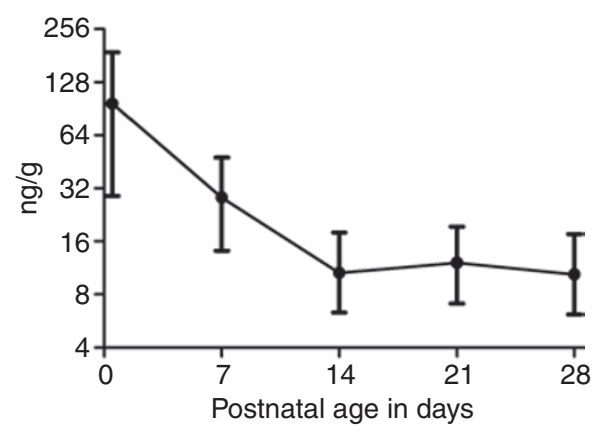

d

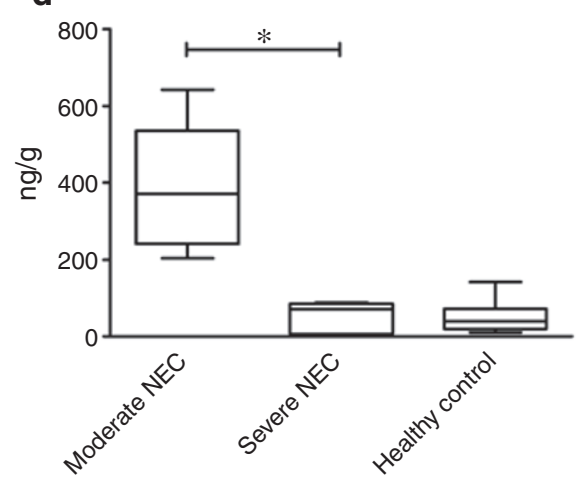

f

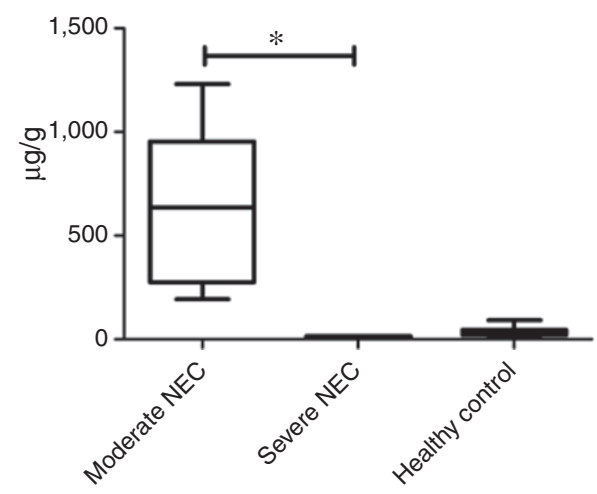

Figure 1. Fecal hBD1 and hBD2 concentrations in ELBW infants. (a) Evolution of fecal hBD2 concentrations in healthy premature infants $(n=56)$. Meconium analysis excludes patients with clinical chorioamnionitis. The number of available fecal samples were 44,43 , and 24 for days 1,14 , and 28 , respectively. (b) Evolution of fecal hBD1 concentrations in healthy premature infants $(n=56)$. Meconium analysis excludes patients with clinical chorioamnionitis. The number of available fecal samples were 44, 43, and 24 for days 1, 14, and 28, respectively. (c) Fecal hBD2 concentrations $12-72 \mathrm{~h}$ before first clinical diagnosis of NEC. Numbers of patients were six with moderate NEC, six with severe NEC, and 24 control patients. Numbers of fecal samples analyzed per group were 14, 15, and 34, respectively. Mean numbers of samples per patient were $2.3,2.5$, and 1.4 , respectively. All infants at least provided at least one sample and none more than three. (d) Fecal hBD2 concentrations at onset of NEC stage 2 plus. Numbers of patients were six with moderate NEC, six with severe NEC, and 24 control patients. Each patient provided one fecal sample. (e) Fecal calprotectin concentrations $12-72 \mathrm{~h}$ before first clinical diagnosis of NEC. Numbers of patients were six with moderate NEC, six with severe NEC, and 24 control patients. Numbers of fecal samples analyzed per group were 14,15 , and 34, respectively. Mean numbers of samples per patient were $2.3,2.5$, and 1.4, respectively. All infants provided at least one sample and none more than three. (f) Fecal calprotectin concentrations at onset of NEC stage 2 plus. Numbers of patients were six with moderate NEC, six with severe NEC, and 24 control patients. Each patient provided one fecal sample. NEC was defined according to the modified Bell's criteria. Data are expressed as the mean \pm 2 SD. Significance testing was performed using one-way ANOVA and values for $P<0.05$ were considered to be statistically significant. ${ }^{*} P<0.001$. ELBW, extremely low birth weight; $h B D$, human $\beta$-defensin; NEC, necrotizing enterocolitis.

onset of NEC (Figure 1d). By contrast, in infants with moderate NEC, fecal hBD2 concentrations further increased to $385 \mathrm{ng} / \mathrm{g}$ (204-642 ng/g, $P=0.009$ ), reaching levels as high as $1,084 \mathrm{ng} / \mathrm{g}$ during disease progression. Similarly, fecal calprotectin concentrations were exclusively elevated in patients with moderate NEC before $(274 \mu \mathrm{g} / \mathrm{g}$, range $63-486)$ and at clinical onset of disease (643 $\mu \mathrm{g} / \mathrm{g}$, range 238-1,047), whereas ELBW infants with severe NEC did not display any increase in fecal calprotectin concentrations before or at clinical onset of NEC (Figure 1e,f). No changes of fecal hBD1 concentrations were observed in relation to NEC. 


\section{Articles | Jenke et al.}

Using immunohistochemistry, we compared hBD2 protein levels in intestinal samples obtained during surgical intervention (i.e., bowel resection) in infants with severe $\operatorname{NEC~}(n=6)$ with samples obtained from those ELBW infants who survived severe NEC $(n=2)$ when they had their stoma closure at term. In accordance with our findings in fecal samples, hBD2 expression was very low during acute NEC (Figure 2a), whereas the same patients showed substantial hBD2 expression several weeks later during colostomy closure (Figure 2b). Here, hBD2 expression was mainly located in close proximity to the base of small intestinal crypts (Figure $2 \mathrm{~b}$ ). During acute NEC, the expression pattern of hBD2 did not differ between survivors and nonsurvivors (data not shown).

In the same surgical resection specimens, we analyzed hBD2, IL-8, and villin (a marker for epithelial cell content) mRNA using real-time PCR (RT-PCR). As control groups, we included intestinal biopsy samples obtained from a cohort of 16 healthy children (age mean 12.1 y, 9 females) without any histological changes and inflamed mucosal biopsies from 26 children with Crohn's disease (age mean 12.6 y, 15 females). No significant differences in villin expression were observed between patients with severe NEC as compared with healthy children or patients with Crohn's disease. In contrast, mRNA copy numbers of the inflammatory cytokine IL- 8 were found to be 10-fold higher in ELBW infants with severe NEC as compared with healthy children but not as high as in patients with Crohn's disease, reflecting the degree of mucosal inflammation (Figure 3a). Of note, despite significantly increased IL-8 levels in ELBW infants with severe NEC, hBD2 levels were 50- to 100 -fold lower as compared with those of inflamed distal small-bowel samples obtained from children with active Crohn's disease (Figure 3a).

\section{Intraindividual Expression of hBD2, TLR4, and MD2}

We next analyzed mRNA expression levels of mucosal hBD2, TLR4, and MD2 in intestinal resection material of two ELBW infants who survived severe NEC. Samples were obtained during acute NEC and $14 \mathrm{wk}$ later at term during stoma closure. Here, we observed 10-fold higher mRNA levels for hBD2 in samples taken during stoma closure as compared with active NEC (Figure 3c). Using conventional RT-PCR, MD2 and TLR4 mRNAs were not detectable in intestinal samples taken during NEC, whereas nested PCR yielded products in all samples (Figure 3b). For semiquantitative analysis, we therefore amplified MD2 and TLR4 mRNA using conventional PCR before RT-PCR with nested primers. Results were normalized for $\beta$-actin and glyceraldehyde 3-phosphate dehydrogenase. As demonstrated in Figure 3c, mRNA levels were about threefold higher for MD2 and fivefold higher for TLR4 at stoma closure as compared with the levels during acute NEC.

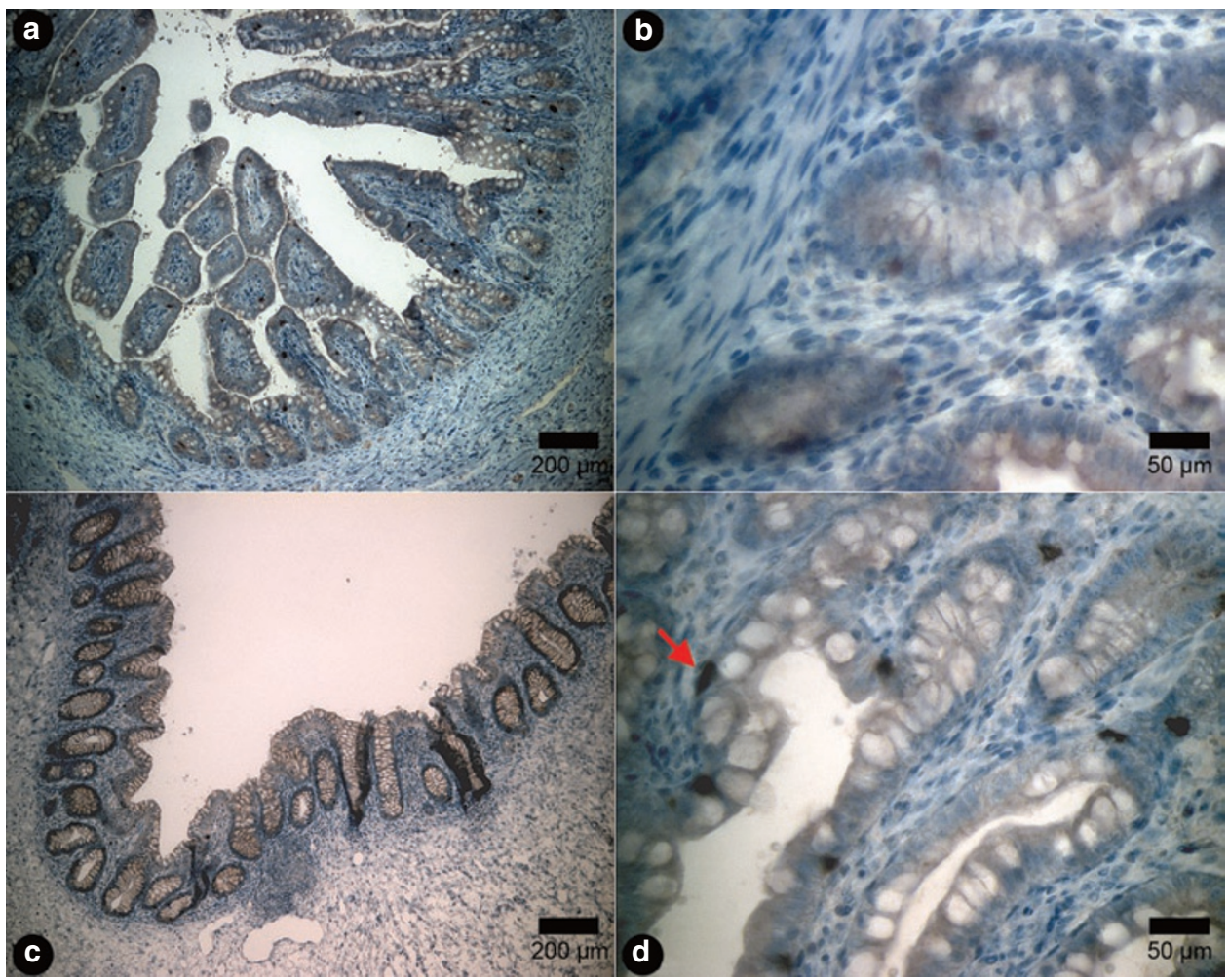

Figure 2. hBD2 expression in the healthy and inflamed intestine of ELBW infants with severe necrotizing enterocolitis (NEC). (a,b) hBD2-positive cells (brown) were almost absent in paraffin sections of the intestines of patients with severe NEC. Samples shown have been obtained from the distal small bowel of two ELBW infants who survived NEC. In (c) jejunum and (d) ileum obtained at term during stoma closure from ELBW infants with previous severe NEC ( $n=2)$, hBD2-expression is observed in cells located in the crypts of Lieberkühn (red arrow). Samples shown correspond to the two patients shown in (a) and (b). Presented images are representative for all sections analyzed. Original magnification in (a) and (c) was $\times 100$ and in (b) and (d) was $\times 400$. ELBW, extremely low birth weight; hBD, human $\beta$-defensin. 
a

1

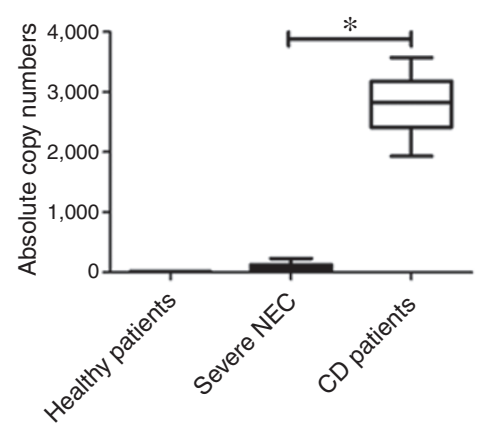

b

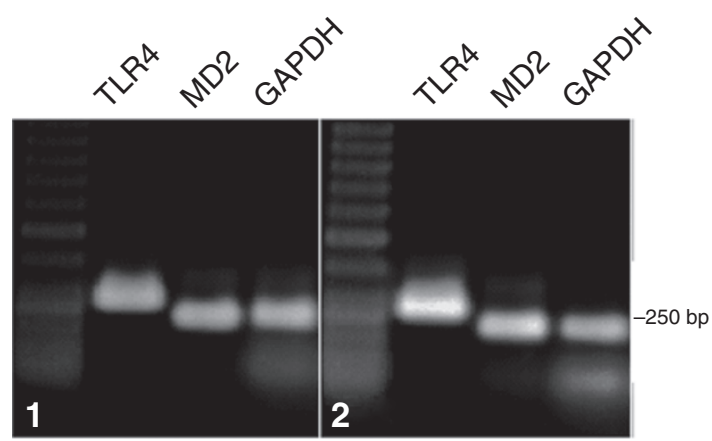

2

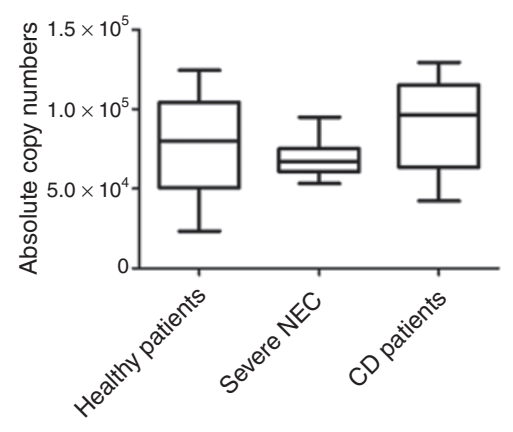

\section{3}

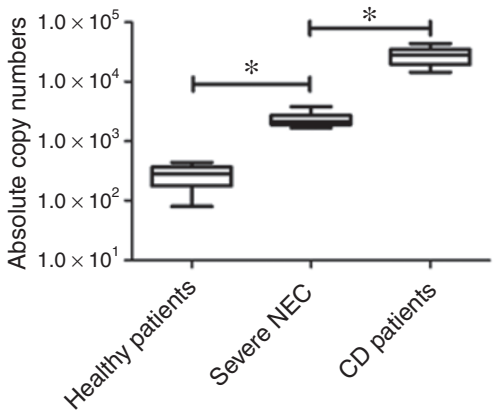

C

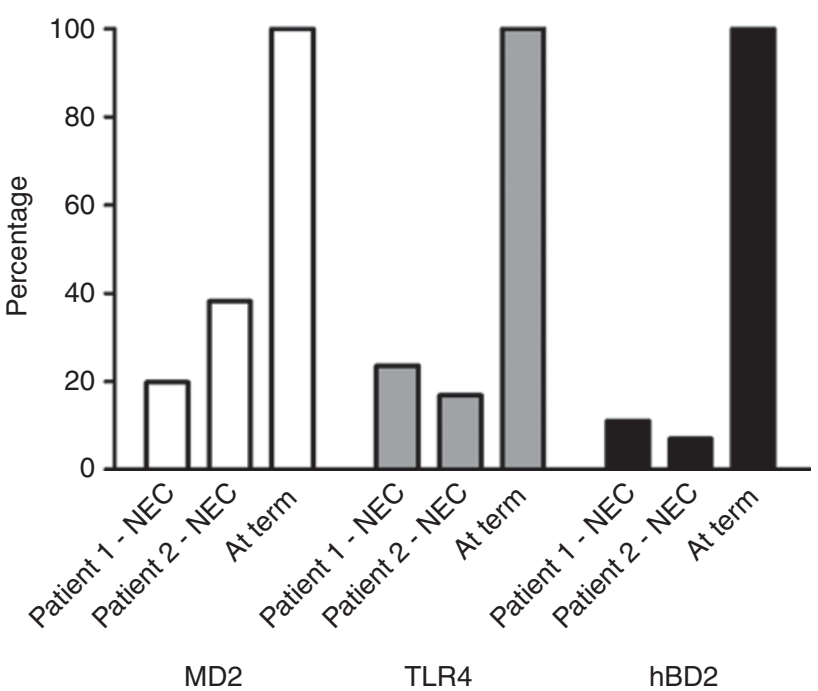

Figure 3. mRNA analysis of hBD2, interleukin-8 (IL-8), villin, MD2, and TLR4. (a) Expression of hBD2 (1), IL-8 (2), and villin (3) in ELBW infants with severe NEC $(n=6)$ compared with children with active Crohn's disease $(n=26)$ and healthy controls $(n=16)$. (b) MD2, TLR4, and GAPDH expression were detected by nested PCR in intestinal samples of patients during severe NEC (1) and during stoma closure (2). (c) Intraindividual analysis of hBD2, MD2, and TLR4 expression during severe NEC and at term during stoma closure of two individual patients. Values obtained at term have been set to $100 \%$. All samples were normalized against the geometric mean of two reference genes (those encoding GAPDH and $\beta$-actin). For all experiments measurements were done in triplicate. Data are expressed as the mean \pm SD of absolute mRNA copy numbers. Values for $P<0.05$ were considered to be statistically significant. * $P$ value $<0.001$. CD, Crohn's disease; ELBW, extremely low birth weight; GAPDH, glyceraldehyde 3-phosphate dehydrogenase; hBD, human $\beta$-defensin; NEC, necrotizing enterocolitis; TLR4, Toll-like receptor 4.

\section{DISCUSSION}

NEC is one of the most serious conditions in ELBW infants associated with substantial morbidity and mortality. Despite extensive research, our understanding of its pathogenesis is still limited. In this study, we investigated the role and regulation of the antimicrobial peptides hBD1 and hBD2 during and after NEC in a cohort of ELBW infants and matched healthy controls. We showed that fecal hBD2 concentrations in infants with moderate NEC were elevated before clinical onset of symptoms, probably reflecting an adequate innate intestinal immune response. If confirmed in a larger cohort, this could potentially allow early diagnosis and intervention, ultimately leading to a better outcome. However, this finding is largely limited by the fact that fecal hBD2 levels in ELBW infants who eventually developed severe NEC showed no increase before and during disease. Thus, hBD2 is more an additional explanatory variable improving our concept of disease pathogenesis in NEC rather than an early predictive or diagnostic marker.

Of note, in addition to the observed low hBD2 levels, patients with severe NEC also showed no increase in fecal calprotectin concentrations, suggesting a specific lack of innate defense activation rather than an impaired intestinal epithelial barrier. Hence, low hBD2 expression is unlikely to be a consequence of a decreased viability or number of intestinal epithelial tissues. This hypothesis is further supported by the fact that we did not observe any differences in the expression of villin, which is known to function as an antiapoptotic protein (16) and to be reduced in epithelial cell loss due to dextran sodium sulfateinduced colitis or inflammatory bowel diseases $(17,18)$.

As an alternative hypothesis to explain low fecal calprotectin concentration, an impaired timely recruitment of granulocytes to the epithelial barrier should be considered. This is 
particularly interesting given the fact that hBD2 functions as a chemotactic factor for human neutrophils (19). Of note, analyzing hBD2 levels $14 \mathrm{wk}$ later (at term) during stoma closure in severely affected ELBW infants who survived, we found significantly increased levels, demonstrating a generally intact hBD2 expression capacity. Taken together, these data suggest that an impaired induction of hBD2 as part of an immature intestinal innate immune defense may play an important role particularly in NEC as ELBW infants with an increase in fecal hBD2 concentrations showed a much more moderate course of the disease. This hypothesis is in line with several reports demonstrating an impaired innate and adaptive immune response in ELBW infants $(1,2,20)$.

As hBD2 has been shown to be at least partly regulated by TLR4 and MD2 signaling (10,21), we investigated expression levels of these receptor molecules during and $14 \mathrm{wk}$ after NEC in the two surviving patients. Concordantly to hBD2, we found expression levels for MD2 and TLR4 to be significantly decreased in the distal small bowel of ELBW infants during NEC as compared with the same patients several weeks later at term during stoma closure.

Despite the descriptive nature of our study and the limited patient number, our findings are in strong agreement with the results presented by Wolfs et al., who demonstrated complete absence of MD2 in the healthy and inflamed premature gut, suggesting an impaired LPS sensing as an important factor involved in the development of NEC in premature infants (13). It is also in line with the low expression profile for TLR4 described in monocytes of ELBW infants as compared with term infants (22). In contrast, conflicting data come from recent observations made in a mouse model where NEC is induced by formula feeding, hypoxia, and LPS exposure. In this model, TLR4 stimulation seemed to reduce enterocyte proliferation (23) and overexpression of TLR4 would hence increase the risk of developing epithelial barrier dysfunction and-in case of prematurity-NEC. Accordingly, C3H/HeJ mice carrying malfunctioning TLR4 develop significantly less gastrointestinal inflammation and seem to be protected against NEC (12). However, a recent study in a similar model using C57BL/6 mice carrying malfunctioning TLR4 in which intestinal injury and inflammation were induced by ischemia alone without LPS exposure reported a protective effect of TLR4 (24), demonstrating that experimental animal models might only partly reflect the very complicated situation of NEC in human premature infants. Given the contradicting evidence from earlier reports and the low number of patients included in this part of our study, no final conclusion can be reached. Nevertheless, we believe that our results underline the importance of further studies focusing on the innate immune system with the aim of eventually defining its exact role in the pathophysiology of NEC.

We also analyzed the dynamics of postnatal fecal hBD2 levels in healthy ELBW infants. Here, we observed very high hBD2 concentrations in meconium, which most likely reflect maternal hBD2 secreted into amniotic fluid and swallowed by the fetus as the levels were within the same range as reported for the amniotic fluid of pregnant women $(25,26)$. Moreover, in our study, these reports also demonstrated positive correlation of amniotic hBD2 levels with premature rupture of membranes and microbial invasion of the amniotic cavity $(25,26)$. During the first $2 \mathrm{wk}$ of life, fecal hBD2 concentrations dropped, reaching a nadir at $\sim 2 \mathrm{wk}$ and slowly increasing from there on showing a similar postnatal dynamic as in two previous smaller studies $(9,27)$. Concentrations of fecal hBD1 were similarly high in meconium and dropped afterwards to a very low baseline in all patients, which is consistent with several studies demonstrating the developmental regulation of hBD1 with almost no expression before $24 \mathrm{wk}$ of gestational age $(28,29)$. hBD1 is highly expressed in breast milk (30), and this possibly contributes to the beneficial effect of breast feeding in prevention of NEC in ELBW infants.

In summary, this study demonstrates that ELBW infants with induced hBD2 expression on NEC have a much more moderate course of the disease as compared with infants without induced hBD2 expression. This provides further evidence for the role of an immature innate immune system in contributing to the development of NEC and its severity in ELBW infants. The exact mechanism(s) causing the observed phenomenon remain unclear; however, other aspects of the intestinal epithelial barrier such as TLR4 are likely to be involved. Further studies are required to follow up this promising lead.

\section{METHODS}

Ethical approval was obtained from the ethics committee of Witten/ Herdecke University for all participating hospitals and full written informed consent was obtained from parents or legal guardians. The study was performed under consideration of the Declaration of Helsinki with amendments from Tokyo 1975, Edinburgh 2000, and Seoul 2008.

\section{Patient Cohort and Sample Collection}

Patients for this study were recruited over a period of 21 mo (April 2008 to December 2009) from three neonatal intensive care units (Wuppertal, Schwerin, and Erfurt). All preterm infants with a gestational age of up to $27+0 \mathrm{wk}$ and born weighing $<1,000 \mathrm{~g}$ were included in this study. Gestational age was determined based on fetal ultrasound before the 14th wk of gestation. Stool samples were prospectively collected on alternate days for the first $4 \mathrm{wk}$ of life. Immediately after collection, samples were stored for a maximum of $48 \mathrm{~h}$ at $4^{\circ} \mathrm{C}$ and then stored at $-80^{\circ} \mathrm{C}$. To verify the stability under such conditions, we measured hBD1 and hBD2 concentrations immediately after sampling and after $48 \mathrm{~h}$ storage at $4{ }^{\circ} \mathrm{C}$ followed by $2 \mathrm{wk}$ at $-80^{\circ} \mathrm{C}$ in the same samples. A total of 20 samples were analyzed and variance was found to be $<10 \%$. The stability of fecal calprotectin has been demonstrated earlier (31). In addition, epidemiological parameters and data about the hospital course were collected daily. This included, for example, medication, type of diet, and amount of enteral feeding. If gastrointestinal surgery due to NEC became necessary, full-thickness intestinal samples from the distal small bowel (in areas viable, not macroscopically necrotic) were taken and stored for later analysis. NEC was classified according to the modified Bell's classification (32). Clinical chorioamnionitis was defined as maternal white blood cell count $>20 / \mathrm{nl}$, temperature $>38^{\circ} \mathrm{C}$, and C-reactive protein $>2 \mathrm{mg} / \mathrm{dl}$ in the absence of any other infection, and histopathological signs of acute chorioamnionitis.

After the recruitment phase, all ELBW infants who progressed to $\mathrm{NEC} \geq$ stage $3 \mathrm{a}$ within $24 \mathrm{~h}$ of symptom onset were identified and classified as having severe NEC. ELBW infants with NEC $\geq$ stage 2 a were classified as having moderate NEC, and patients who never developed NEC during their hospital course were classified as the control group. Patients with spontaneous intestinal perforation were excluded from the study. Spontaneous intestinal perforation was defined at laparotomy as the presence of an isolated intestinal perforation surrounded by normal-appearing bowel and the absence of characteristic gross or 
microscopic features of NEC. Infants in the control group with any of the following diagnoses were excluded: any other surgery, severe bronchopulmonary dysplasia (stage 3 ), retinopathy of prematurity ( $\geq$ stage 3 ), significant intraventricular hemorrhages ( $\geq$ stage 3 ), as these diseases significantly alter clinical management in particular considering enteral and parenteral nutrition.

As additional control cohorts we included 28 noninflamed terminal ileum biopsies from healthy children, and 32 inflamed samples from children with Crohn's disease, which were obtained in a previous study (7).

\section{Calprotectin, hBD2, and hBD1 Analysis in Fecal Samples}

Fecal calprotectin (Immundiagnostik, Bennsheim, Germany), hBD2 (Immundiagnostik), and hBD1 (Alpha Diagnostic, San Antonio, TX) concentrations were determined by enzyme-linked immunosorbent assays as described earlier (9,33). Extraction of antimicrobial peptides from stool samples was performed according to the manufacturer's recommendations. Briefly, a $100 \mathrm{mg}$ fecal sample was weighed and diluted in an urea- and citrate-containing buffer in a weight:volume ratio of 1:50. After vortex for $30 \mathrm{~s}$ followed by centrifugation for $5 \mathrm{~min}$ at $13,000 \mathrm{rpm}$, supernatants were immediately subjected to enzymelinked immunosorbent assay. All analyses were performed in triplicate and normalized against controls provided by the manufacturers. Intraand interassay coefficients of variation were $<5 \%$ and $<15 \%$, respectively. Laboratory personnel were blinded to the origin and identity of patient samples.

\section{Immunohistochemistry}

Paraffin-embedded formalin-fixed sections were treated twice for $10 \mathrm{~min}$ in xylene and subsequently incubated twice in 100,85 , and $70 \%$ ethanol to remove paraffin. Antigen retrieval was performed in Tris/EDTA ( $\mathrm{pH}$ 9.0). Slides were then subjected to standard immunohistochemistry. Rabbit anti-hBD2 (C2) was used as primary antibody (kindly donated by Tomas Ganz, UCLA, Los Angeles, CA). Indirect visualization was performed using secondary antirabbit antibodies (Jackson ImmunoResearch Europe, Newmarket, UK) conjugated with horseradish peroxidase. Cell nuclei were stained using hematoxylin. Tissue sections were analyzed using a Leica DM RB transmission light microscope equipped with a Leica DC300F CCD camera (Leica Microsystems, Wetzlar, Germany).

\section{RNA Extraction and Reverse Transcription PCR}

Paraffin-embedded formalin-fixed sections were treated as described earlier to remove paraffin. After standard RNA extraction using Trizol reagent (Invitrogen, Carlsbad, CA) integrity of extracted RNA was analyzed by agarose gel electrophoresis and quantified using spectrophotometric absorbance at $260 \mathrm{~nm}$. Thereafter, $500 \mathrm{ng}$ of RNA were reverse transcribed using a QIAGEN QuantiFast kit (QIAGEN, Hilden, Germany).

\section{Real-Time (RT)-PCR and Absolute Quantification of hBD2, VIL1, and IL-8 mRNA Copy Numbers}

RT-PCR analysis was performed on a Rotor Gene 6000 real time rotary analyzer (Corbett Life Science/QIAGEN, Hilden, Germany) using SYBR green methodology as described earlier (7). All values were normalized against the geometric mean of two reference genes (those encoding glyceraldehyde 3-phosphate dehydrogenase and $\beta$-actin) as described earlier (34). For amplification, Taq-Polymerase from Fermentas (Glen Burnie, MD) was used with an error rate of $0.22 \times 10^{-4}$. All primers were synthesized by Biomers (Ulm, Germany).

\section{Analysis of mRNA Levels of MD2 and TLR4 by RT-PCR}

For relative intraindividual quantification of MD2 and TLR4 mRNA, we used a nested PCR approach. After reverse transcription, MD2 and TLR4 were first amplified using the following primers: MD2_for 5'-CCTGTTTTCTTCCATATTTACTG-3', MD2_rev 5'-CTTGAAGGAGAATGATATTGTTG-3', TLR4_for 5'-TGCATGG AGCTGAATTTCTAC-3', and TRL4_rev 5'-TGAGCCACATTAAG TTCTTTC-3'

Cycling conditions were as follows: $10 \mathrm{~min}$ at $95^{\circ} \mathrm{C}$, followed by 35 cycles at $95^{\circ} \mathrm{C}$ for $30 \mathrm{~s}, 58^{\circ} \mathrm{C}$ for $40 \mathrm{~s}$, and $72^{\circ} \mathrm{C}$ for $30 \mathrm{~s}$.
Second-round RT-PCR analysis was performed using SYBR green methodology using the following primers: MD2nested_for 5'-TGGGTCTGCACCTCATCCG-3',MD2nested_rev5'-AATAACTT CTTTGCGCTTTGG-3', TLR4nested_for 5'-ACCTGGACCTGAGC TTTAATC-3', and TLR4nested_rev 5'-CCAATGGGGAAGTTCT CTAG-3'

Cycling conditions were as follows: $10 \mathrm{~min}$ at $95^{\circ} \mathrm{C}$, followed by 40 cycles at $95^{\circ} \mathrm{C}$ for $20 \mathrm{~s}$ and $60^{\circ} \mathrm{C}$ for $40 \mathrm{~s}$. mRNA levels were analyzed in relation to each other after normalization against the geometric mean of two reference genes (those encoding glyceraldehyde 3-phosphate dehydrogenase and $\beta$-actin) as described earlier.

\section{Statistical Analysis}

Mean expression levels of all genes tested were obtained from triplicate RT-PCR measurements. Data are presented as the mean \pm SD. Testing for significant differences between groups was performed using a one-way ANOVA test for values with Gaussian distribution and Mann-Whitney test for values without Gaussian distribution. The Kolmogorov-Smirnov test was used to rule out non-Gaussian distribution. Values for $P<0.05$ were considered statistically significant. All analyses were performed using GraphPad version 5.01 (GraphPad Software, La Jolla, CA). Fecal samples were not available for each individual patient at each time point. Cases with missing values were excluded from analysis and differences in case numbers due to unavailable fecal samples are specifically mentioned in the corresponding tables and figures.

\section{ACKNOWLEDGMENTS}

The authors thank Claudia Foerster as well as Silvia Vogel (technicians) for their excellent technical support. hBD2 antibodies were kindly donated by Tomas Ganz (UCLA). All authors contributed significantly to this work: A.C.W.J. and J.P. were involved in study design, experimental analysis, coordination, and data interpretation. M.Z. and S.W. were involved in study design, coordination, and data interpretation.

\section{STATEMENT OF FINANCIAL SUPPORT}

This study was funded by the European Society of Paediatric Infectious Diseases and the HELIOS Research Center.

Disclosure: The authors declared no conflict of interest.

\section{REFERENCES}

1. Levy O, Martin S, Eichenwald E, et al. Impaired innate immunity in the newborn: newborn neutrophils are deficient in bactericidal/permeabilityincreasing protein. Pediatrics 1999;104:1327-33.

2. Schelonka RL, Infante AJ. Neonatal immunology. Semin Perinatol 1998;22:2-14.

3. Lin PW, Stoll BJ. Necrotising enterocolitis. Lancet 2006;368:1271-83.

4. Blakely ML, Tyson JE, Lally KP, et al. Laparotomy versus peritoneal drainage for necrotizing enterocolitis or isolated intestinal perforation in extremely low birth weight infants: outcomes through 18 months adjusted age. Pediatrics 2006;117:e680-7.

5. Gewolb IH, Schwalbe RS, Taciak VL, Harrison TS, Panigrahi P. Stool microflora in extremely low birthweight infants. Arch Dis Child Fetal Neonatal Ed 1999;80:F167-73.

6. Huttner KM, Bevins CL. Antimicrobial peptides as mediators of epithelial host defense. Pediatr Res 1999;45:785-94.

7. Zilbauer M, Jenke A, Wenzel G, et al. Expression of human beta-defensins in children with chronic inflammatory bowel disease. PLoS ONE 2010; 5:e15389.

8. Schröder JM, Harder J. Human beta-defensin-2. Int J Biochem Cell Biol 1999;31:645-51.

9. Richter M, Topf HG, Gröschl M, et al. Influence of gestational age, cesarean section, and type of feeding on fecal human beta-defensin 2 and tumor necrosis factor-alpha. J Pediatr Gastroenterol Nutr 2010;51:103-5.

10. Gariboldi S, Palazzo M, Zanobbio L, et al. Low molecular weight hyaluronic acid increases the self-defense of skin epithelium by induction of beta-defensin 2 via TLR2 and TLR4. J Immunol 2008;181:2103-10.

11. Visintin A, Mazzoni A, Spitzer JA, Segal DM. Secreted MD-2 is a large polymeric protein that efficiently confers lipopolysaccharide sensitivity to Toll-like receptor 4. Proc Natl Acad Sci USA 2001;98:12156-61. 
12. Leaphart CL, Cavallo J, Gribar SC, et al. A critical role for TLR4 in the pathogenesis of necrotizing enterocolitis by modulating intestinal injury and repair. J Immunol 2007;179:4808-20.

13. Wolfs TG, Derikx JP, Hodin CM, et al. Localization of the lipopolysaccharide recognition complex in the human healthy and inflamed premature and adult gut. Inflamm Bowel Dis 2010;16:68-75.

14. Sadeghi K, Berger A, Langgartner M, et al. Immaturity of infection control in preterm and term newborns is associated with impaired toll-like receptor signaling. J Infect Dis 2007;195:296-302.

15. Josefsson S, Bunn SK, Domellöf M. Fecal calprotectin in very low birth weight infants. J Pediatr Gastroenterol Nutr 2007;44:407-13.

16. Khurana S, George SP. Regulation of cell structure and function by actinbinding proteins: villin's perspective. FEBS Lett 2008;582:2128-39.

17. Kersting S, Bruewer M, Schuermann G, et al. Antigen transport and cytoskeletal characteristics of a distinct enterocyte population in inflammatory bowel diseases. Am J Pathol 2004;165:425-37.

18. Wang Y, Srinivasan K, Siddiqui MR, George SP, Tomar A, Khurana S. A novel role for villin in intestinal epithelial cell survival and homeostasis. J Biol Chem 2008;283:9454-64.

19. Niyonsaba F, Ogawa H, Nagaoka I. Human beta-defensin-2 functions as a chemotactic agent for tumour necrosis factor-alpha-treated human neutrophils. Immunology 2004;111:273-81.

20. Splawski JB, Jelinek DF, Lipsky PE. Delineation of the functional capacity of human neonatal lymphocytes. J Clin Invest 1991;87:545-53.

21. Varoga D, Klostermeier E, Paulsen F, et al. The antimicrobial peptide HBD- 2 and the Toll-like receptors- 2 and -4 are induced in synovial membranes in case of septic arthritis. Virchows Arch 2009;454:685-94.

22. Sadeghi K, Berger A, Langgartner M, et al. Immaturity of infection control in preterm and term newborns is associated with impaired toll-like receptor signaling. J Infect Dis 2007;195:296-302.

23. Sodhi CP, Shi XH, Richardson WM, et al. Toll-like receptor-4 inhibits enterocyte proliferation via impaired beta-catenin signaling in necrotizing enterocolitis. Gastroenterology 2010;138:185-96.
24. Tatum PM Jr, Harmon CM, Lorenz RG, Dimmitt RA. Toll-like receptor 4 is protective against neonatal murine ischemia-reperfusion intestinal injury. J Pediatr Surg 2010;45:1246-55.

25. Soto E, Espinoza J, Nien JK, et al. Human beta-defensin-2: a natural antimicrobial peptide present in amniotic fluid participates in the host response to microbial invasion of the amniotic cavity. J Matern Fetal Neonatal Med 2007;20:15-22.

26. Iavazzo C, Tassis K, Gourgiotis D, et al. The role of human beta defensins 2 and 3 in the second trimester amniotic fluid in predicting preterm labor and premature rupture of membranes. Arch Gynecol Obstet 2010;281:793-9.

27. Campeotto F, Baldassarre M, Laforgia N, et al. Fecal expression of human $\beta$-defensin-2 following birth. Neonatology 2010;98:365-9.

28. McCray PB Jr, Bentley L. Human airway epithelia express a beta-defensin. Am J Respir Cell Mol Biol 1997;16:343-9.

29. Starner TD, Agerberth B, Gudmundsson GH, McCray PB Jr. Expression and activity of beta-defensins and LL-37 in the developing human lung. J Immunol 2005;174:1608-15.

30. Jia HP, Starner T, Ackermann M, Kirby P, Tack BF, McCray PB Jr. Abundant human beta-defensin-1 expression in milk and mammary gland epithelium. J Pediatr 2001;138:109-12.

31. Naess-Andresen CF, Egelandsdal B, Fagerhol MK. Calcium binding and concomitant changes in the structure and heat stability of calprotectin (L1 protein). Clin Mol Pathol 1995;48:M278-84.

32. Kliegman RM, Walsh MC. Neonatal necrotizing enterocolitis: pathogenesis, classification, and spectrum of illness. Curr Probl Pediatr 1987;17:213-88.

33. Schröder O, Naumann M, Shastri Y, Povse N, Stein J. Prospective evaluation of faecal neutrophil-derived proteins in identifying intestinal inflammation: combination of parameters does not improve diagnostic accuracy of calprotectin. Aliment Pharmacol Ther 2007;26:1035-42.

34. Vandesompele J, De Preter K, Pattyn F, et al. Accurate normalization of real-time quantitative RT-PCR data by geometric averaging of multiple internal control genes. Genome Biol 2002;3:RESEARCH0034. 